\title{
Algorithms for virtual topology reconfiguration under multi-hour traffic using Lagrangian relaxation and Tabu Search approaches
}

\author{
R. Aparicio-Pardo, P. Pavon-Marino, N. Skorin-Kapov*, B. Garcia-Manrubia, J. Garcia-Haro \\ Technical University of Cartagena, (UPCT), Plaza del Hospital 1, 30202, Cartagena, Spain \\ * Faculty of Electrical Engineering and Computing (FER), University of Zagreb, Unska 3, Zagreb, Croatia \\ Tel: (34) 968 325952,Fax: (34) 968 325973, e-mail: pablo.pavon@upct.es
}

\begin{abstract}
Periodic reconfiguration of the virtual topology in transparent optical networks has been recently investigated as a mechanism to more efficiently adapt the network to predictable periodic traffic variations along the day or week. The scheduling of periodic reconfigurations should consider the trade-off between a lower network cost obtained through better resource allocation, and the undesired traffic disruptions that these reconfigurations may cause. This paper presents and compares two algorithms for planning virtual topology reconfiguration suitable for exploring this trade-off. The first is based on a Lagrangian relaxation of the planning problem, and the second is based on a Tabu Search Tabu Search meta-heuristic. The merits of both algorithms are compared.
\end{abstract}

Keywords: virtual topology design, network planning, multi-hour traffic, Lagrangian relaxation, Tabu search.

\section{INTRODUCTION}

Transparent optical networks based on Wavelength Division Multiplexing (WDM) have become an accepted solution for new broadband backbone networks [1]. In these networks, traffic is carried through transparent alloptical connections, called lightpaths. A lightpath is established between an optical transmitter in the originating node and an optical receiver in the terminating node over a wavelength channel in each traversed fibre. Since the traffic carried over a lightpath is wavelength switched at intermediate nodes, the costs associated to the data electronic processing are saved. The set of lightpaths established onto a physical topology to support a given set of traffic demands (in Gbps) is called a virtual topology. The Virtual Topology Design (VTD) problem implies solving a two-layered routing problem: in the upper layer, it involves routing traffic flows (demands) over the virtual topology; while in the lower layer, it involves finding a Routing and Wavelength Assignment (RWA) [2] of lightpaths over the physical topology. In this work, we assume network scenarios with an over-provisioned fiber plant where a feasible RWA solution exists for almost any lightpath in the virtual topology, focusing only on the upper layer routing problem.

The VTD problem under multi-hour or periodic traffic is denoted as Multi-Hour Virtual Topology Design (MH-VTD) [3]. In this case, the set of traffic demands are represented as a temporal sequence of traffic matrices, reflecting traffic variations over a period of time (e.g. a day or a week). The MH-VTD problem can be classified into: (i) the MH-VTD-NR (non-reconfigurable) problem, where a time-fixed (non-reconfigurable) virtual topology is planned to carry the traffic demand at any time; (ii) the MH-VTD-R (reconfigurable) problem where a time-varying (reconfigurable) virtual topology is designed to more efficiently adapt to known (or estimated) traffic variations. In both problems classes, we consider the number of optical transceivers in the network as the measure of the network cost to optimize. The present work is focused on the MH-VTD-R problem where, as a second optimization criterion, the number of reconfigurations associated with the evolution of the virtual topology is minimized.

We conclude this introduction by summarizing the previous studies related to the present work. Multi-hour network design has been researched for multiple network technologies [3]-[7] (see [4] for a comprehensive survey). In the last decade, the interest of the optical community has shifted to lightpath-based transparent optical networks [3], [4]-[7]. Most research efforts have been focused on planning in the lower layer, i.e., RWA of a given sequence of virtual topology designs corresponding to $\mathrm{MH}$ traffic [5]. However, the present work is focused on the upper layer problem, i.e. determining the evolution of the virtual topology from the MH traffic [3], [6], [7]. Finally, this paper presents two approaches to solve the MH-VTD-R planning problem with penalization on reconfiguration frequency: (i) a Lagrangian Relaxation method and (ii) an enhancement of the Tabu Search Tabu Search algorithm presented in [7] to handle this problem variant.

\section{THE LANGRANGEAN RELAXATION (LR) APPROACH}

Let $N$ be the number of nodes, and $T$ be the number of time intervals. Let $i, j, s, d, n=\{1 \ldots N\}$ be the indices for the nodes, and $t=\{1 \ldots T\}$ be the index for the time intervals (note that $t=T$ is followed by $t=1$ ). Let $m_{s d}{ }^{t}$ denote the traffic demand (measured in Gbps) from node $s$ to node $d$, during time interval $t$. Let $C$ denote the lightpath capacity in Gbps. The cost of each transmitter and receiver is considered equal, and is represented by $c_{l}$. Finally,

The work described in this paper was carried out with the support of the BONE-project ("Building the Future Optical Network in Europe"), a Network of Excellence funded by the European Commission through the $7^{\text {th }}$ ICT-Framework Programme, the support of the MEC Spanish project TEC2007-67966-01/TCM CONPARTE-1, the support of the Fundación Séneca project FORMA 00002/CS/08, and developed in the framework of "Programa de Ayudas a Grupos de Excelencia de la Región de Murcia, de la Fundación Séneca (Plan Regional de Ciencia y Tecnología 2007/2010)"; This work was also partially supported by research projects SAFE, funded by the Unity Through Knowledge Fund in Croatia, and 036-0362027-1641, funded by the Ministry of Science, Education and Sports, Croatia. 
an artificial cost of reconfiguring a lightpath is denoted as $c_{2}$.

\subsection{A MILP formulation for the MH-VTD-R problem with penalization of reconfiguration}

The decision variables of the problem are:

- $\quad \mathbf{f}=\left(f_{i j}^{s d t}\right)=[0,1]$. Fraction of total demand $m_{s d}{ }^{t}$ routed on lightpaths from node $i$ to node $j$ in time $t$.

- $\quad \mathbf{p}=\left(p_{i j}{ }^{t}\right)=\{0,1,2, \ldots\}$. Number of lightpaths from node $i$ to node $j$, required during time interval $t$.

- $\quad \mathbf{t x}=t x_{n}=\{0,1,2, \ldots\}$. Number of transmitters installed in node $n$.

- $\quad \mathbf{r x}=r x_{n}=\{0,1,2, \ldots\}$. Number of receivers installed in node $n$.

- $\quad \mathbf{r}^{+}=\left(r_{i j}^{+}\right)=\{0,1,2, \ldots\}$. Number of new lightpaths set up at time $t$ with respect to the lightpaths at time $t-1$

- $\mathbf{r}^{-}=\left(r_{i j}^{-}{ }^{\dagger}=\{0,1,2, \ldots\}\right.$. Number of lightpaths torn down at time $t$ with respect to the lightpaths at time $t-1$

The problem formulation is given by (1).

$$
\min c_{1} \sum_{n}\left(t x_{n}+r x_{n}\right)+c_{2} \sum_{i, j, t} r^{+t}
$$

subject to:

$$
\begin{gathered}
\sum_{s, d}\left\{m_{s d}^{t} \cdot f_{i j}^{s d t}\right\} \leq C \cdot p_{i j}^{t}, \quad i, j=\{1, \ldots, N\}, t=\{1, \ldots, T\} \\
\sum_{j} f_{n j}^{s d t}-\sum_{i} f_{i n}^{s d t}=\left\{\begin{array}{l}
1, \text { if } n=s \\
-1, \text { if } n=d, n, s, d=\{1, \ldots, N\}, t=\{1, \ldots, T\} \\
0 \text { otherwise }
\end{array}\right. \\
t x_{n} \geq \sum_{j} p_{n j}^{t}, n=\{1, \ldots, N\}, t=\{1, \ldots, T\} \\
r x_{n} \geq \sum_{i} p_{i n}^{t}, n=\{1, \ldots, N\}, t=\{1, \ldots, T\} \\
p_{i j}^{t}-p_{i j}^{t-1}=r^{+t}{ }_{i j}^{t}-r^{-t}{ }_{i j}^{t}, i, j=\{1, \ldots, N\}, t=\{1 \ldots, T\}
\end{gathered}
$$

\subsection{The Lagrangian Relaxation-based Dual Subgradient Optimization Algorithm}

This method was previously applied in [4], [8] to solve multihour problems in other network technologies. In this work, the algorithm is applied to transparent optical networks. Since the general procedure used in this paper is the same described in [4], [8], we refer to these references for a more detailed description. We will focus solely on the distinctive features of this application.

The Lagrangian Relaxed Problem appears when constraints (1b), (1d) and (1e) are "relaxed" (or "dualized") by adding them (weighted by Lagrangian multipliers) to objective function (1a) and eliminating them from the total set of constraints of (1). Let $\boldsymbol{\lambda}=\left(\lambda_{i j}{ }^{\dagger}\right), \boldsymbol{\mu}=\left(\mu_{i}{ }^{\dagger}\right)$ and $\boldsymbol{v}=\left(v_{j}{ }^{\dagger}\right)$ be the Lagrangian (or dual) multipliers associated with constraints (1b), (1d) and (1e), respectively. Then, the Lagrangian function $L\left(\boldsymbol{\lambda}, \boldsymbol{\mu}, \mathbf{v}, \mathbf{f}, \mathbf{p}, \mathbf{t x}, \mathbf{r x}, \mathbf{r}^{+}\right)$ resulting from the relaxation is:

$$
\begin{aligned}
& L(\boldsymbol{\lambda}, \boldsymbol{\mu}, \mathbf{v}, \mathbf{f}, \mathbf{p}, \mathbf{t x}, \mathbf{r x}, \mathbf{r}+)=c_{1} \sum_{n}\left\{t x_{n}+r x_{n}\right\}+c_{2} \sum_{i, j, t} r^{+t}{ }_{i j}+\sum_{i, j, t} \lambda_{i j}^{t} \cdot\left\{\sum_{s, d}\left\{m_{s d}^{t} \cdot f_{i j}^{s d t}\right\}-C \cdot p_{i j}^{t}\right\}+\sum_{i, t} \mu_{i}^{t} \cdot\left\{\sum_{j} p_{i j}^{t}-t x_{i}\right\}+ \\
& +\sum_{j, t} v_{j}^{t} \cdot\left\{\sum_{i} p_{i j}^{t}-r x_{j}\right\}=\sum_{n}\left\{c_{1}-\sum_{t} \mu_{n}^{t}\right\} \cdot t x_{n}+\sum_{n}\left\{c_{1}-\sum_{t} v_{j}^{t}\right\} \cdot r x_{n}+\sum_{t, i, j} \lambda_{i j}^{t} \cdot\left\{\sum_{s, d}\left\{m_{s d}^{t} \cdot f_{i j}^{s d t}\right\}\right\}+c_{2} \sum_{i, j, t} r^{+}{ }_{i j}^{t}+ \\
& +\sum_{t, i, j}\left\{\mu_{i}^{t}+v_{j}^{t}-C \cdot \lambda_{i j}^{t}\right\} \cdot p_{i j}^{t}=L_{1}(\boldsymbol{\mu}, \mathbf{t x})+L_{2}(\mathbf{v}, \mathbf{r} \mathbf{x})+L_{3}(\boldsymbol{\lambda}, \mathbf{f})+L_{4}\left(\boldsymbol{\lambda}, \boldsymbol{\mu}, \mathbf{v}, \mathbf{r}^{+}, \mathbf{p}\right)
\end{aligned}
$$

Minimization of the Lagrangian function $L\left(\boldsymbol{\lambda}, \boldsymbol{\mu}, \mathbf{v}, \mathbf{f}, \mathbf{p}, \mathbf{T}, \mathbf{R}, \mathbf{r}^{+}\right)$(2) can be decoupled into four sets of separate minimization subproblems since the relaxation of constraints (1b), (1d) and (1e) has broken the dependencies among some decision variables in (1). The first and second sets of subproblems are associated to variables $\mathbf{t x}$ and $\mathbf{r x}$, respectively. The third set corresponds to variables $\mathbf{f}$ solely restrained by constraints (1c). And, finally, in the fourth set of subproblems, variables $\mathbf{p}, \mathbf{r}^{+}$and $\mathbf{r}^{-}$remain linked through constraints (1e) and (1f). 


$$
\begin{aligned}
& \min _{\mathbf{f}, \mathbf{p}, \mathbf{t x}, \mathbf{r x}, \mathbf{r}^{+}}\left\{L\left(\boldsymbol{\lambda}, \boldsymbol{\mu}, \mathbf{v}, \mathbf{f}, \mathbf{p}, \mathbf{t x}, \mathbf{r x}, \mathbf{r}^{+}\right) \mid \text {constraints }(1 \mathrm{c}),(1 \mathrm{e}),(1 \mathrm{f})\right\}=\min _{t \mathbf{x}}\left\{L_{1}(\boldsymbol{\mu}, \mathbf{t x})\right\}+\min _{\mathbf{r x}}\left\{L_{2}(\mathbf{v}, \mathbf{r x})\right\}+ \\
& \min \left\{L_{3}(\boldsymbol{\lambda}, \mathbf{f}) \mid \text { constraints }(1 \mathrm{c})\right\}+\min _{\mathbf{p}, \mathbf{r}^{+}}\left\{L_{4}\left(\boldsymbol{\lambda}, \boldsymbol{\mu}, \mathbf{v}, \mathbf{p}, \mathbf{r}^{+} \mid \text {constraints }(1 \mathrm{e}),(1 \mathrm{f})\right\}\right.
\end{aligned}
$$

While the optimal solution $\mathbf{f *}$ to the third subproblem is primal-feasible, that is, feasible from the point of view of the primal problem (1), solutions $\mathbf{p}^{*}, \mathbf{t} \mathbf{x}^{*}, \mathbf{r} \mathbf{x}^{*}$, and $\mathbf{r}^{+*}$ from the remaining subproblems in (5) might not be so. This is because we have relaxed some constraints. Consequently, we must find a total primal-feasible solution starting from $\mathbf{f}^{*}$. First, we use $\mathbf{f}^{*}$ in (1b) to compute a primal-feasible solution $\mathbf{p}^{\mathbf{p f}}$. And, secondly, we repeat the procedure using the solution $\mathbf{p}^{\mathbf{p f}}$ in (1d) and (1e) to derive primal-feasible $\mathbf{t x}^{\mathbf{p f}}$ and $\mathbf{r} \mathbf{x}^{\mathbf{p f}}$, and, again, in (1f) and ( $1 \mathrm{~g})$ to derive primal-feasible $\mathbf{r}^{+\mathbf{p f}}$ and $\mathbf{r}^{\text {-pf }}$.

\section{THE TABU SEARCH TABU SEARCH (TS) APPROACH}

The Tabu Search approach for the MH-VTD-R problem proposed in this paper extends the Tabu Search algorithm from [7] with a second optimization criterion where lightpath reconfigurations are minimized. In [7], in each iteration, the Tabu Search heuristic explores all neighbouring solutions of the current one, except for those prohibited by the tabu list. The search iteratively solves smaller single-time slot MILP formulations ((4) in [7]) with various constraints on transceivers for independent time intervals in order to jump between neighbouring solutions. All neighbouring solutions are evaluated according to a fitness function. The best neighbour becomes the new current solution in the next iteration. We encourage the reader to consult [7] for a more detailed description of the algorithm. Fitness functions for both the MH-VTD-NR and MH-R-VTD-R problems are presented in [7], where minimization of the number of transceivers is the only optimization criterion. Here, we present a third fitness function suitable for the MH-R-VTD-R problem which includes minimization of reconfiguration frequency as a second criterion:

$$
c_{1} \sum_{n}\left\{\max _{t} \sum_{j, j \neq n} p_{n j}^{t}\right\}+c_{1} \sum_{n}\left\{\max _{t} \sum_{i, i \neq n} p_{i n}^{t}\right\}+c_{2} \sum_{i, j, t}\left|p_{i j}^{t}-p_{i j}^{t-1}\right|
$$

We can observe that (7) matches with (1a) expressed in terms of $p_{i j}{ }^{t}$, since solutions constructed by the Tabu Search algorithm in each iteration are derived from single time-interval MILP formulations ((4) in [7]) for all $t$ where decision variables $\mathbf{t x}, \mathbf{r x}, \mathbf{r}^{+}$and $\mathbf{r}^{-}$cannot be defined.

\section{RESULTS}

The Lagrangian Relaxation (LR)-based and the Tabu Search (TS)-based approaches have been tested and compared for two different network traffic data scenarios. In both testing scenarios, the multihour traffic consists of 24 matrices used to illustrate hourly fluctuations over the course of a day. Furthermore, the physical topology spans over several time zones aimed to investigate the temporal mismatch of the most loaded traffic hour.

In the first scenario, we used data from a real traffic trace in the eleven-node Abilene network, publicly available at [9]. This data consists of traffic matrices spanning several weeks. Thus, we averaged all values of the trace taken at the same time in the day to obtain an average daily sequence of 24 matrices.

The second scenario corresponds to synthetic multi-hour traffic, generated for a 15-node worldwide network. The physical topology is described in Table 1 and Fig. 5 from [10]. The traffic was generated using the multihour model proposed in [11], for which the traffic between two nodes is proportional to the product of the two node populations; the variation of the traffic activity along the day in a node evolves according to expression (2) from [11]; and the traffic demand between a pair of nodes depends of the activity of both end nodes.

Once the multihour traffic was generated, the traffic was normalized by multiplying it with a normalization factor $(n f)$. Value $n f$ was calculated such that the average traffic between two nodes in the most loaded time slot is equal to $\rho \cdot C$, where $C$ is the lightpath capacity, and $\rho$ is a traffic load parameter. The values tested in our study were $\rho=\{0.1,1,10\}$. A value of $\rho=0.1$ corresponds to the case when the average traffic between two nodes in the most loaded time slot is only $10 \%$ of a single lightpath capacity. On the contrary, a value of $\rho=10$ captures cases in which the average traffic between two nodes in the most loaded time slot is the capacity of ten lightpaths.

For all the conduced tests, Table 1shows: (i) the lower bounds (LB) on the number of transceivers proposed in [7]; (ii) the number of transceivers and, finally, in brackets: (iii) the number of reconfigurations provided by the two heuristic approaches. Even though the LR approach was run for 1000 iterations, the best solution was reached after approximately 50 iterations for all the experiments. The stopping criterion in the TS algorithm was fixed to 20 iterations without improving the best solution found and the size of the tabu list was set to 7 and 9 for the eleven-node Abilene network and the fifteen-node worldwide network, respectively. The results show that TS achieves better values with respect to the number of transceivers, while the LR performs better with 
respect to reconfiguration frequency. Nevertheless, the good results achieved by the LR in reconfiguration performance should not necessarily be considered as an absolute merit since the additional number of transceivers with respect to the TS algorithm permits solutions with fewer reconfigurations. Furthermore, we can observe that the transceiver performance of the LR versus TS worsens with load parameter $\rho$, while the reconfiguration performance improves. On the other hand, with respect to optimality of the solutions in terms of the number of transceivers, comparison of the heuristic results with the lower bound indicates that the TS is able to provide good approximate solutions for loads $\rho=1$ and $\rho=10$ (even quasi-optimal solutions for $\rho=10$ ). However, for a load of $\rho=0.1$, it is difficult to conclude whether the lower bound is weak or the heuristic solutions are far from optimal, as the suboptimal gap is not negligible.

Table 1.The total number of transceivers (and reconfigurations) obtained for both testing scenarios.

\begin{tabular}{c|c|c|c|c}
\hline & P & LB & TS & LR \\
\hline & 0.1 & 36 & $52(201)$ & $93(40)$ \\
Abilene Network & 1 & 248 & $260(219)$ & $369(97)$ \\
& 10 & 2385 & $2392(1078)$ & $2497(1048)$ \\
\hline \multirow{3}{*}{ Worlwide Network } & 0.1 & 66 & $104(499)$ & $290(210)$ \\
& 1 & 506 & $516(653)$ & $774(232)$ \\
& 10 & 4920 & $4924(3331)$ & $5134(3092)$ \\
\hline
\end{tabular}

\section{CONCLUSIONS}

In this paper, two heuristic algorithms based on a Langrangean Relaxation and a Tabu Search approach are proposed to solve the Reconfigurable Multi-Hour Virtual Topology Design (MH-VTD-R) problem assuming two optimization criterions. Both the number of optical transceivers required at the nodes and the number of lightpath reconfigurations necessary to handle the traffic variations over time are minimized. Several test cases are conducted to compare the performance of the two proposed algorithms. The results reveal the superiority of the Tabu Search approach with respect to transceiver cost, but at a trade-off with increased reconfiguration frequency.

\section{REFERENCES}

[1] J. Berthold, A. A. M. Saleh, L. Blair, and J. M. Simmons: Optical Networking: Past, Present, and Future, IEEE Journal of Lightwave Technology, vol. 26, no. 9, pp.1104-1118, May 2008.

[2] H. Zang, J. P. Jue, and B. Mukherjee: A Review of Routing and Wavelength Assignment Approaches for Wavelength-Routed Optical WDM Networks, Optical Network Magazine, pp. 47-59, Jan. 2000.

[3] P. Pavon-Marino, R. Aparicio-Pardo, B. Garcia-Manrubia, and N. Skorin-Kapov, "Virtual topology design and flow routing in optical networks under multihour traffic demand," Photonic Network Communications, vol. 19, no. 1, pp. 42-54, Feb. 2010.

[4] M. Pioro and D. Medhi: Routing, Flow and Capacity Design in Communication and Computer Networks. San Francisco, CA: Morgan Kaufmann, 2004, pp. 455-474.

[5] J. Kuri, N. Puech, M. Gagnaire, E. Dotaro, and R. Douville: Routing and wavelength assignment of scheduled lightpath demands, IEEE Journal on Selected Areas in Communications, vol. 21, pp. 12311240, Oct. 2003.

[6] F. Ricciato, S. Salsano, A. Belmonte, and M. Listanti: Off-line Configuration of a MPLS over WDM Network under Time-Varying Offered Traffic, IEEE Infocom, pp. 57-65, 2002.

[7] N. Skorin-Kapov, P. Pavon-Marino, B. Garcia-Manrubia, and R. Aparicio-Pardo: Scheduled Virtual Topology Design Under Periodic Traffic in Transparent Optical Networks, in Proc. 6th ICST International Conference on Broadband Communications, Networks and Systems, BroadNets 2009, Madrid, Spain, Sept. 2009.

[8] D. Medhi and D. Tipper: Some approaches to solving a multihour broadband network capacity design problem with single-path routing, Telecommunication Systems, vol. 13, no. 2-4, pp. 269-291, Jul. 2000.

[9] TOTEM Project: Toolbox for Traffic Engineering Methods [Online]. Available: http://totem.run.montefiore.ulg.ac.be/datatools.html

[10] P. Pavon-Marino, R. Aparicio-Pardo, B. Garcia-Manrubia, and J. Garcia-Haro, WDM networks planning under multi-hour traffic demand with the MatPlanWDM tool, in Proc. Industry Track "Simulation Works" collocated with 1st International ICST Conference on Simulation Tools and Techniques for Communications, Networks and System, Marseille, France, Mar. 2008.

[11] J. Milbrandt, M. Menth, S. Kopf: Adaptive Bandwidth Allocation: Impact of Traffic Demand Models for Wide Area Networks, in 19th International Teletraffic Congress (ITC19), Beijing, China, 2005. 